\title{
An in situ tissue engineering scaffold with growth factors combining angiogenesis and osteoimmunomodulatory functions for advanced periodontal bone regeneration
}

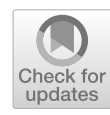

Tian Ding, Wenyan Kang, Jianhua Li, Lu Yu and Shaohua Ge*

\begin{abstract}
Background: The regeneration of periodontal bone defect remains a vital clinical challenge. To date, numerous biomaterials have been applied in this field. However, the immune response and vascularity in defect areas may be key factors that are overlooked when assessing the bone regeneration outcomes of biomaterials. Among various regenerative therapies, the up-to-date strategy of in situ tissue engineering stands out, which combined scaffold with specific growth factors that could mimic endogenous regenerative processes.

Results: Herein, we fabricated a core/shell fibrous scaffold releasing basic fibroblast growth factor (bFGF) and bone morphogenetic protein-2 (BMP-2) in a sequential manner and investigated its immunomodulatory and angiogenic properties during periodontal bone defect restoration. The in situ tissue engineering scaffold (iTE-scaffold) effectively promoted the angiogenesis of periodontal ligament stem cells (PDLSCS) and induced macrophage polarization into pro-healing M2 phenotype to modulate inflammation. The immunomodulatory effect of macrophages could further promote osteogenic differentiation of PDLSCs in vitro. After being implanted into the periodontal bone defect model, the iTE-scaffold presented an anti-inflammatory response, provided adequate blood supply, and eventually facilitated satisfactory periodontal bone regeneration.
\end{abstract}

Conclusions: Our results suggested that the iTE-scaffold exerted admirable effects on periodontal bone repair by modulating osteoimmune environment and angiogenic activity. This multifunctional scaffold holds considerable promise for periodontal regenerative medicine and offers guidance on designing functional biomaterials.

Keywords: Periodontal bone regeneration, In situ tissue engineering, Angiogenesis, Osteoimmunomodulation, Biomimetic repair

*Correspondence: shaohuage@sdu.edu.cn

Department of Periodontology \& Biomaterials, School and Hospital of Stomatology, Cheeloo College of Medicine, Shandong University \& Shandong Provincial Key Laboratory of Oral Tissue Regeneration \& Shandong Engineering Laboratory for Dental Materials and Oral Tissue Regeneration, Jinan 250012, China

\begin{abstract}
Background
Periodontitis is a worldwide epidemic inflammatory disease that results in the progressive destruction of periodontal tissues, including connective tissue attachment and alveolar bone [1]. And periodontal bone destruction caused by periodontitis is regarded as one of the major reasons for tooth loss, which has adverse effects on mastication and aesthetics [2]. Unfortunately, the existing therapies for periodontitis, including initial periodontal therapy, flap surgery, and guided bone regeneration,
\end{abstract} original author(s) and the source, provide a link to the Creative Commons licence, and indicate if changes were made. The images or other third party material in this article are included in the article's Creative Commons licence, unless indicated otherwise in a credit line to the material. If material is not included in the article's Creative Commons licence and your intended use is not permitted by statutory regulation or exceeds the permitted use, you will need to obtain permission directly from the copyright holder. To view a copy of this licence, visit http://creativecommons.org/licenses/by/4.0/. The Creative Commons Public Domain Dedication waiver (http://creativeco mmons.org/publicdomain/zero/1.0/) applies to the data made available in this article, unless otherwise stated in a credit line to the data. 
can only control disease progression while has limited capacity for bone repair [3]. The current treatments cannot completely repair the damaged periodontal bone owing to the sole focus on osteogenesis [4]. Actually, bone regeneration is a sequence of closely coordinated and overlapping processes, including inflammatory response, angiogenesis, and remodeling, which contains a lot of sophisticated cell types and signal molecules [5]. It is widely believed that immune response immediately after injury and plays a crucial part in the entire process of wound healing $[6,7]$. And angiogenesis is indispensable for bone homeostasis and repair since new blood vessels not only bring nutrients but act as the pathway for inflammatory cells, mesenchymal stem cells (MSCs), and bone precursor cells to support injured bone repair $[8,9]$. Thus, it is still of great scientific value to pay attention to immunomodulation and vascularization during the initial process of periodontal bone repair.

In situ tissue engineering strategy, which utilizes biomaterials to deliver multiple cytokines to take advantage of the host regenerative potential and mimic endogenous regenerative processes, has been regarded as a hopeful technology for periodontal bone defect therapy [10, 11]. Natural wound repair is a multi-step process that is orchestrated by multiple cytokines in an appropriate extracellular matrix microenvironment. This procedure can be intelligibly separate into early inflammation/angiogenesis and late tissue remodeling. Hence, appropriate combination of cytokines and release at optimal time points may be beneficial to bone tissue regeneration. Basic fibroblast growth factor (bFGF), the first discovered prototypical member of FGF family, acts as a pleiotropic part in cellular and metabolic homeostasis [12]. It is an essential growth factor (GF) in wound healing because of its benefits as a powerful inducer of vascularization [13], proliferation [14], and migration [15]. Recently, Pan et al. discovered that bFGF could reduce the production of pro-inflammatory factors and promoted lipopolysaccharide (LPS)-stimulated inflammatory macrophages polarization towards anti-inflammatory M2 phenotype through AKT/P38/NF-kB signaling pathways [16]. Another study reported that bFGF could suppress LPSmediated inflammatory responses in periodontal ligament stem cells (PDLSCs) ex vivo [17]. Therefore, bFGF is frequently employed to rapid resolution of inflammatory responses in the tissue and promote endogenous stromal cells recruitment, proliferation, and angiogenesis for bone repair. After controlling inflammation, neovascularization, and obtaining enough cells, the later phase of tissue remodeling should be activated. Bone morphogenic protein-2 (BMP-2), one of the transforming growth factor beta (TGF- $\beta$ ) superfamily members, is a highly potent osteoinductive GF [18]. BMP-2 could boost the formation of Sharpey's fibers, induce MSCs to differentiate into osteoblasts, and accelerate the regeneration and repair process of bone tissues [19]. Previously, our group has reported an in situ tissue engineering scaffold (iTEscaffold) and found that application of bFGF followed by BMP-2 could significantly promote periodontal bone regeneration by facilitating stem cell homing, proliferation, and osteogenic differentiation [20,21]. Because the events of immunomodulation and angiogenesis are prior to the differentiation of MSCs-osteoblast lineage during the process of bone healing, it is indispensable to explore osteoimmunomodulatory and angiogenic properties of iTE-scaffold. To the best of our knowledge, this is the first study on osteoimmunomodulatory and angiogenic effects of iTE-scaffold during periodontal bone repair.

Herein, we continue to construct a favorable iTEscaffold for sequential delivery of bFGF and BMP-2 with the coaxial electrospinning technique. The fibrous scaffold was constructed with a poly (lactide-co-glycolide)/ poly (L-lactic acid) (PLGA/PLLA) shell/core structure. PLLA was designed as the inner material due to its longer degradation rate, while PLGA was defined as the sheath owing to its adjustable and comparatively faster degradation rate. We systematically investigated the physicochemical properties and cell compatibility of the fibrous scaffolds. The osteoimmunomodulatory and angiogenic properties of iTE-scaffold were further investigated in vitro. Subsequently, the immunomodulatory function and angiogenic effect of iTE-scaffold on periodontal bone regeneration were characterized using an in vivo rat periodontal bone defect model.

\section{Results and discussion}

\section{Characterization of the fibrous scaffolds}

The scaffold without GFs-loading, was defined as pristine scaffold (P-scaffold), while iTE-scaffold represented the scaffold with BMP-2 in PLLA core and bFGF in PLGA shell. To characterize the structure and surface morphology of different scaffolds, the scaffolds were visualized under scanning electron microscopy (SEM) at different magnifications. As shown in Fig. 1a, all the scaffolds were composed of randomly arranged fibers with interconnected 3D reticulated structure. Inserts of Fig. 1a were photographs of scaffolds. Although, the mean diameters of iTE-scaffold were slightly thicker than that of the P-scaffold (1198.44 nm vs $1235.27 \mathrm{~nm}$ ). However, no statistical difference was found between their diameters $(P>0.05)$. This phenomenon revealed that the incorporation of GFs hardly changed the diameter of fibers. The fluorescence microscope was used to corroborate the presence of shell/core structure within the scaffold. As shown in Additional file 1: Fig. S1, the core and shell showed distinct red and green fluorescence, respectively, 


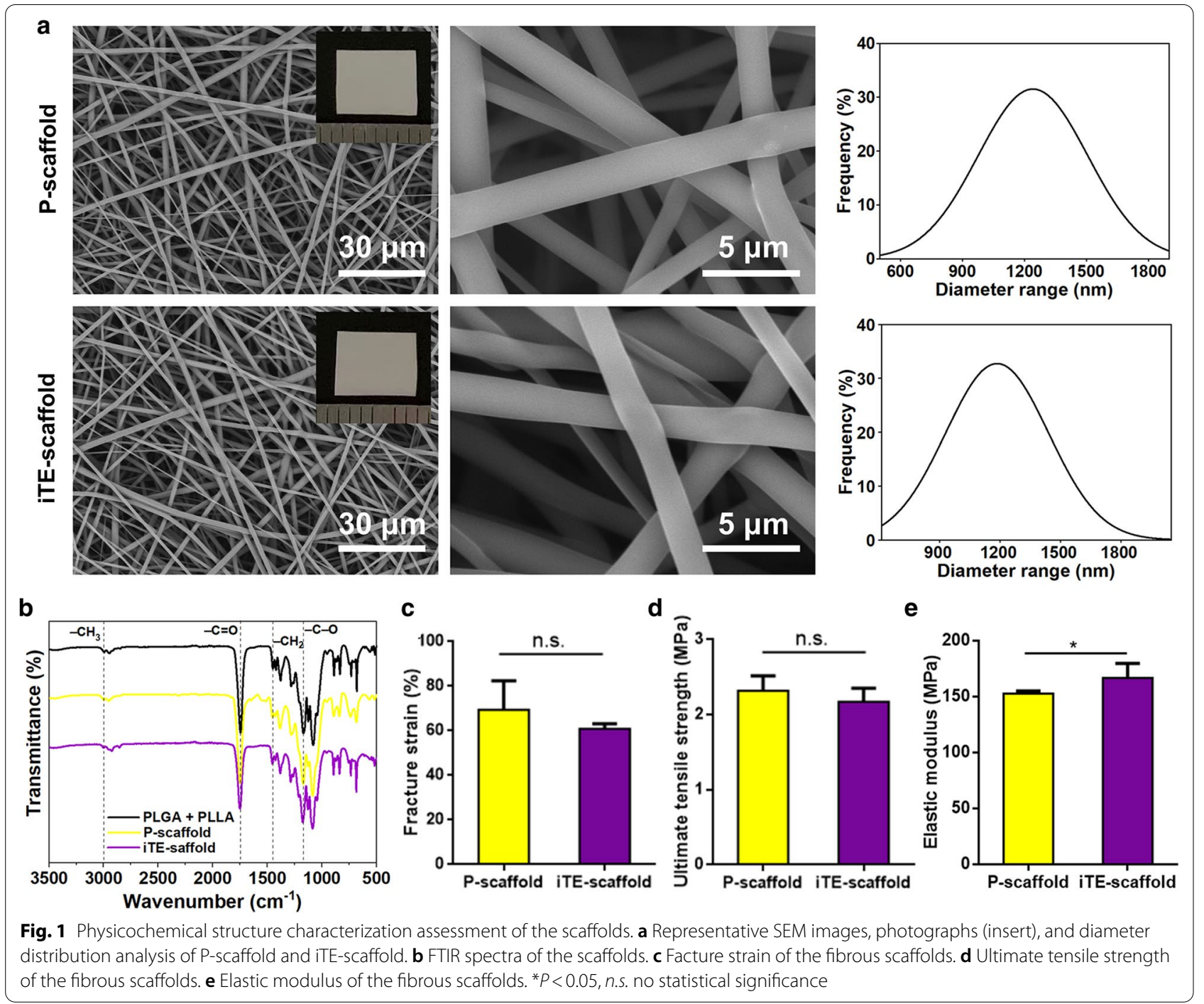

which demonstrated that all the fibrous scaffolds had typical core/sheath structures.

The surface chemical property plays a vital role in cellular regulation. Fourier transform infrared (FTIR) was conducted to evaluate the surface chemical properties and characteristic functional groups of PLLA and PLGA mixture, P-scaffold, and iTE-scaffold (Fig. 1b). As displayed by the classical spectrum of PLLA and PLGA mixture, the frequency at $2997 \mathrm{~cm}^{-1}$ was assigned to $\mathrm{CH}_{3}$ vibration. And the peak at $1451 \mathrm{~cm}^{-1}$ was due to $-\mathrm{CH}_{2}$ bending vibration. In addition, the strong peak at $1752 \mathrm{~cm}^{-1}$ was corresponding to the absorption by $-\mathrm{C}=\mathrm{O}$ and the frequencies at $1172 \mathrm{~cm}^{-1}$ belonged to $-\mathrm{C}-\mathrm{O}$ stretching vibration [22]. The characteristic spectra of P-scaffold and iTE-scaffold resembled those of PLLA and PLGA mixture, and these results revealed that the electrospinning process had no significant effect on the chemical properties, excluded the influence of the diversity of material surface properties on cellular regulation [23].

It's known to us all that the basic function of tissue engineering scaffolds is to provide adequate mechanical support for cell adhesion, growth, and tissue regeneration. Thus, the mechanical performance of the mats was evaluated by tensile testing (Fig. 1c-e). The fracture strain decreased slightly from $74.34 \%$ to $65.92 \%$ by the addition of GFs, and the ultimate tensile strength reduced a little from 3.32 $\mathrm{MPa}$ to $2.77 \mathrm{MPa}$. However, compared to P-scaffold group, the iTE-scaffold presented higher elastic modulus (152.76 MPa vs $166.73 \mathrm{MPa}$ ), and this phenomenon might be due to the incorporation of GFs diminished the polymer crystallinity. This finding suggested that loading with GFs toughened the scaffold, which was in line with former research. The possible 
reason was that the addition of GFs not only augmented the polymer crystallinity, but also enhanced interfacial combination between inorganic and organic phases [24]. Consequently, the iTE-scaffold can meet the standard of sufficient mechanical load capacity of tissue engineering scaffolds according to He et al's standard [25].

In addition, surface wettability is one of nonnegligible factors in the evaluation criteria of the biomaterials for cell adhesion and growth. Besides, Hotchkiss et al. found that macrophages cultured on hydrophilic surface exhibited more potential to differentiate towards anti-inflammatory M2 phenotype [26]. Unsurprisingly, the water contact angle (WCA) measurement results indicated that the iTE-scaffold was significantly more hydrophilic than P-scaffold, which was mostly due to the larger number of hydrophilic bioactive factors presented on the surface of fibrous mat (Additional file 1: Fig. S2). Our results were similar to a previous study, which showed that loading cytokines could ameliorate the hydrophilicity of scaffolds [18]. Consequently, as expected, GFs loading could make the iTE-scaffold more biocompatible. The release profiles of iTE-scaffold displayed a sequential release pattern, which was similar to our previous finding [20]. In brief, bFGF exhibited an initial burst release profile (over 70\%) during the first few days. After that, the releasing rate of bFGF significantly slowed down and reached a standstill. On the contrary, around 4\% of BMP- 2 was released at the same time, because of the protection of the shell, whereas the remaining BMP-2 released continuously in the following 30 days.

\section{Cytocompatibility of the fibrous scaffolds}

The cytotoxicity of the fibrous scaffolds was assessed by live/dead staining and cell counting kit-8 (CCK-8) assay at preset time point. As shown in Fig. 2a, b, there were almost no dead cells on P-scaffold and iTE-scaffold, suggesting the scaffolds had no adverse effect on cells. Subsequently, PDLSCs attachment on the different substrates was statistically analyzed by the determination of cell seeding density $\left(\mathrm{cells} / \mathrm{cm}^{2}\right)$ with respect to the density of PDLSCs initially seeded $\left(2 \times 10^{4}\right.$ cells $\left./ \mathrm{cm}^{2}\right)$. Cells cultured on the tissue culture plate were served as negative control (NC). The above results indicated that cell cultured on the scaffolds exhibited high cell seeding efficiency and excellent cell adhesion similar to those on the NC (Fig. 2c). CCK-8 result was shown in Fig. 2d, and there was no statistical difference among all groups $(P>0.05)$, which further proved that the superior biocompatibility of the scaffolds. As shown in Additional file 1: Fig. S3, macrophages adhered tightly to the scaffolds with pseudopods. Interestingly, cells on the iTE-scaffold exhibited a relatively slender shape. And morphological changes in macrophages caused by topographical cues could further affect their polarization state. It has been reported that pro-healing M2 phenotype is associated with an increase in cell elongation [27]. This finding confirmed the results of surface wettability, and further verified that iTE-scaffold was in favor of tissue regeneration. Therefore, it could be seen that the fibrous scaffolds had ideal biocompatibility and could serve as an appropriate microenvironment for cell adherent and growth.

\section{In vitro angiogenic assessment}

Angiogenesis is an indispensable step for bone healing, due to nutrients brought by vascular networks are essential for cells within the repair tissues, and inadequate angiogenesis can lead to cell death or undesirable tissue integration $[8,9]$. The released bFGF is a strong angiogenic factor [28, 29]. Herein, the angiogenic ability of PDLSCs on different scaffolds was characterized by tube formation assay, quantitative real-time polymerase chain reaction (qRT-PCR), and immunofluorescent staining. PDLSCs cultured in endothelial cell growth medium-2 (EGM-2) were regarded as the $\mathrm{NC}$, while human umbilical vein endothelial cells (HUVECs) resuspended in EGM-2 were regarded as the positive control. For tube formation assay, the iTE-scaffold group exhibited elevated angiogenic ability as showed by dramatically higher network formation parameters compared to control and P-scaffold groups. A significantly higher number of nodes, junctions, meshes, and total tube length were observed in PDLSCs cultured on iTE-scaffold at 12 h (Fig. 3a). Notably, only iTE-scaffold and HUVEC groups still remained the tubular networks, in contrast, the capillary-like networks of NC or P-scaffold groups already collapsed into spheroids after $24 \mathrm{~h}$ seeding on the Matrigel (Fig. 3b). In addition, the expression of angiogenic genes including CD31, vascular endothelial growth factor (VEGF), stem cell factor (SCF), and placental growth factor (PLGF) was further evaluated by qRT-PCR (Fig. 3c). Consistent with the results of tube formation test, the relative expression level of the angiogenic genes in PDLSCs cultured on iTEscaffold was remarkably up-regulated compared to the other groups. Notably, compared to NC group, CD31 mRNA expression of iTE-scaffold group was over 5 times greater. $C D 31$ is an important marker of vascular endothelial differentiation [30], and these results indicated the strong effect of iTE-scaffold on endothelial lineage induction. And endothelial cells played a vital role in keeping the physiological functions of blood vessels in the developed vasculatures [31]. To further validate the results, immunofluorescence staining was adopted to measure the expression level of CD31. As shown in Fig. 3d, more CD31-positive cells were 


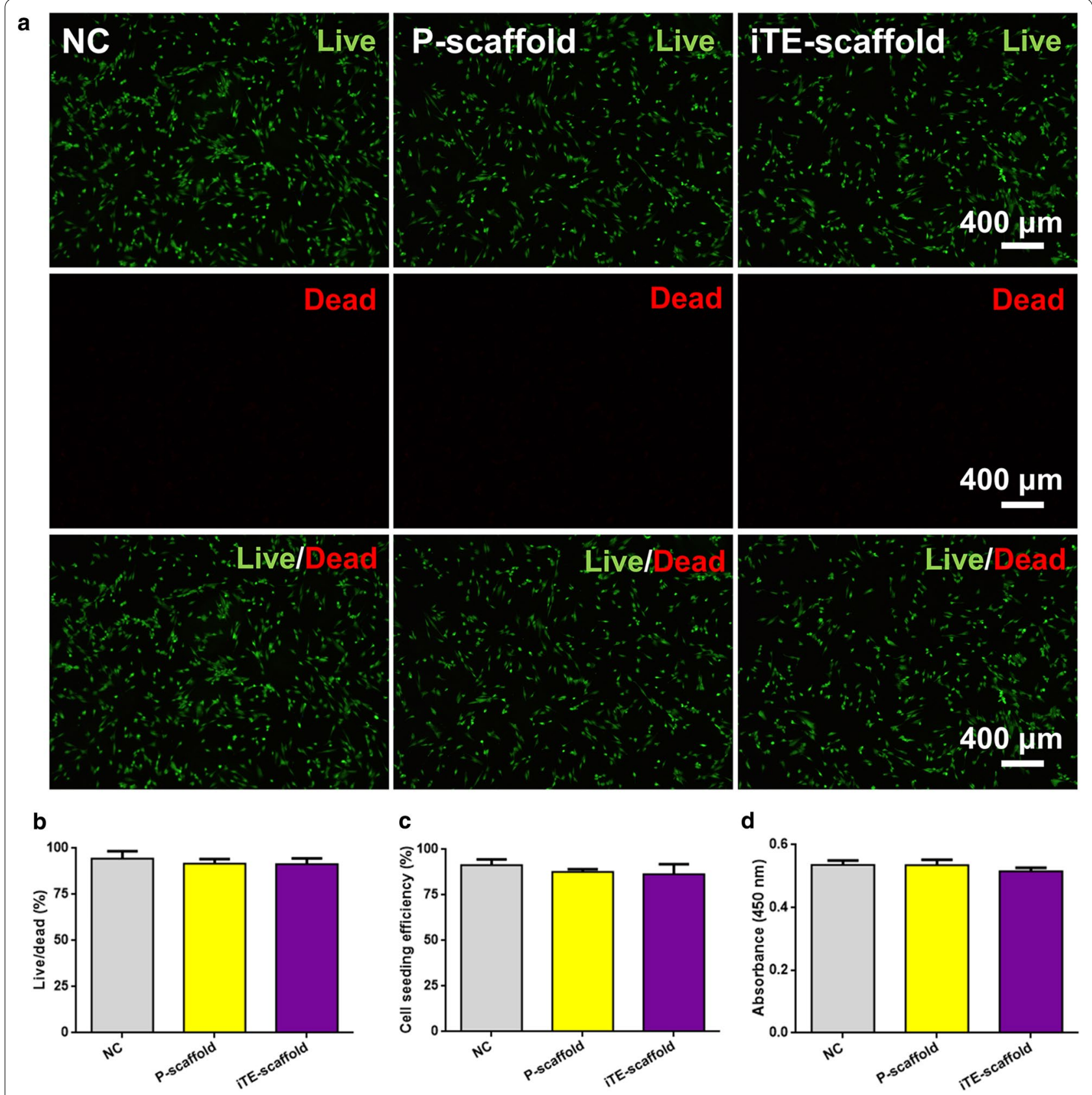

Fig. 2 Cytocompatibility study of the scaffolds. a, b Representative images and quantitative analysis of live/dead staining of PDLSCs incubated on the different substrates. $\mathbf{c}$ Cell seeding effciency of PDLSCs cultured on the different substrates. $\mathbf{d}$ Cell activity on the different substrates

presented in iTE-scaffold group, and there was no significant difference between $\mathrm{NC}$ and P-scaffold groups $(P>0.05)$. Collectively, all above results illuminated that iTE-scaffold maintained its angiogenic capability possibly due to the release of bFGF, and iTE-scaffold was beneficial to vascularization ex vivo, which could provide the basis for periodontal bone regeneration.

\section{In vitro osteoimmunomodulatory function}

\section{characterization}

It is commonly accepted that immune regulation is the first response that occurs after implantation and plays a vital role throughout the entire osteointegration process $[7,32]$. Therefore, endowing biomaterials with admirable osteoimmunomodulatory function provides a new 


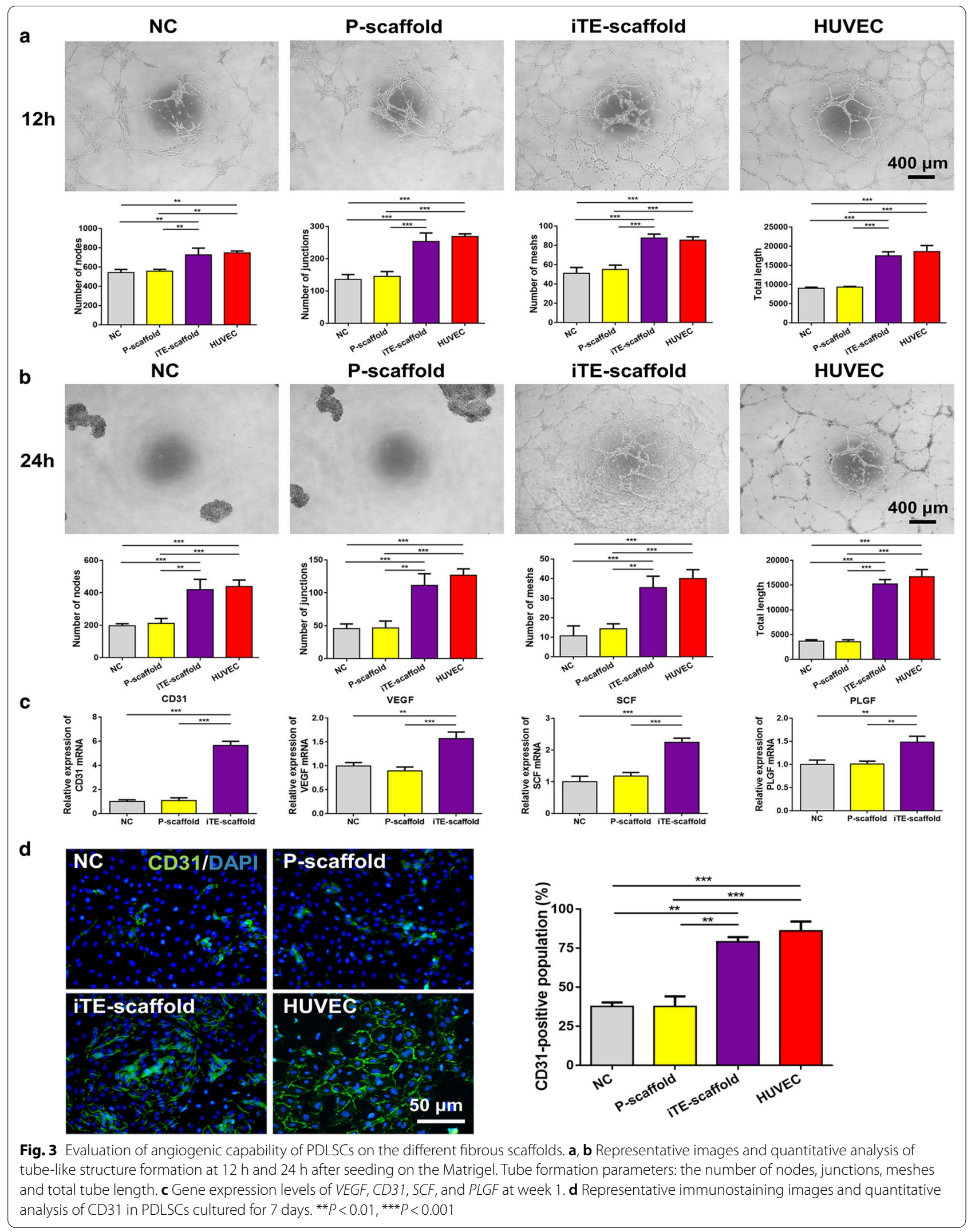


option to improve endogenous bone regeneration by regulating the immune homeostasis [33, 34]. Macrophages, a central regulator of immune defense during tissue healing, can be mainly divided into two phenotypes: classically M1 activated pro-inflammatory phenotype and alternatively M2 anti-inflammatory phenotype. This discrepancy is mainly due to the highly plastic property of macrophages, which could flexibly switch from one phenotype to the other in response to microenvironment signals [23]. It has been reported that the dynamic transition from M1 to M2 macrophages has a profound effect on wound repair $[35,36]$. Here, we studied the effects of the iTE-scaffold on RAW264.7 macrophage polarization and the osteogenic differentiation of PDLSCs. Macrophages were treated with LPS as a simple in vitro stimulus to mimic the inflammatory microenvironment in the process of wound repair [37]. Inducible nitric oxide synthase (iNOS, an M1 phenotype marker) and CD206 (an M2 phenotype marker) were employed to characterize the macrophage phenotypes. Subsequently, we investigated the M1 and M2 phenotype distribution by immunofluorescence staining and flow cytometry. And macrophages cultivated in 10\% fetal bovine serum (FBS) in Dulbecco's modified Eagle's medium (DMEM) without LPS denoted as the NC group, while those cultured in the inflammatory induction medium served as the LPS group. Immunofluorescence staining indicated that LPS treatment significantly increased the $\mathrm{iNOS}^{+}$M1like subpopulation, which was remarkably blunted by treatment with iTE-scaffold (Fig. 4b). Meanwhile, the proportion of $\mathrm{CD}^{206^{+}}$M2-like subpopulation was significantly up-regulated in iTE-scaffold group but unchanged in the other groups. As shown in Fig. 4c, the flow cytometry analysis result was consistent with immunofluorescence staining. In order to further explore the effect of iTE-scaffold on macrophages, qRT-PCR analysis was carried out. iTEscaffold significantly down-regulated the expression level of M1 macrophage-related genes, including tumor necrosis factor (TNF)- $\alpha$, iNOS and interleukin (IL)-1 $\beta$, whereas up-regulated the expression of pro-healing genes that related to anti-inflammatory M2 macrophages such as arginase-1 (Arg-1), CD206 and IL-10, indicating that iTE-scaffold played a vital role in macrophages polarization (Fig. 4d). In addition, macrophages had a great impact on the microenvironment of bone repair sites by cytokine secretion, which both regulated inflammation and controlled cell differentiation [35]. Hence, the levels of pro and anti-inflammatory cytokines secreted by the treated macrophages were detected by enzyme-linked immunosorbent assay (ELISA) (Fig. 4f). Obviously, LPS remarkably promoted the release of pro-inflammatory factors (TNF- $\alpha$ and IL-1 $\beta$ ), which were dramatically higher than those in the NC group. iTE-scaffold abolished the cytokine storms and distinctly stimulated the secretion of pro-healing factors (IL-10 and TGF- $\beta$ ), suggesting a better bone repair potential of the iTE-scaffold. It was reported that bFGF dampened the pro-inflammatory factors production and converted LPS-stimulated inflammatory macrophages polarization into M2 phenotype through AKT/P38/NF-kB pathway [16]. Furthermore, P-scaffold had no significant effect on reversing LPS-induced M1 macrophages and secretion of inflammatory factors. Collectively, these results demonstrated iTE-scaffold stimulated the transition of macrophages towards the M2 type.

To further study the osteoimmunomodulatory effect of the fibrous scaffold, the formally conditional medium of macrophages was collected to culture PDLSCs for 7 days. qRT-PCR analysis was performed to test the gene expression levels of osteogenic markers [alkaline phosphatase $(A L P)$, runt-related transcription factor 2 (Runx2), osteocalcin $(O C N)$, and osteopontin $(O P N)]$ to determine the indirect effect of macrophages on the osteogenic differentiation of PDLSCs. As shown in Fig. 4g, the relative mRNA level of $A L P$ in the iTE-scaffold dramatically up-regulated over threefold compared to that in the NC group. ALP is a vital early phenotype marker during osteogenic differentiation of cells [38]. And Runx2 expression, an important transcription factor regulating lots of other osteogenesis-related genes [39], was significantly upregulated in cells grown in condition medium collected from macrophages cultured on iTE-scaffold. Moreover, $O C N$ and $O P N$ expression was higher than the NC group, which might be due to OPN and $O C N$ were mainly active during the bone matrix mineralization stage [40,41]. These data indicated that iTE-scaffold regulated macrophages could remarkably promote the osteogenic differentiation of PDLSCs. In addition, the expression patterns of all osteogenic genes were significantly inhibited in the LPS and P-scaffold

\footnotetext{
(See figure on next page.)

Fig. 4 Evaluation of the osteoimmunomodulatory function in vitro. a Schematic illustration of biomaterial-mediated macrophage polarization. b Fluorescent staining images of the macrophage phenotypes and quantitative analysis of the CD206/iNOS-positive population. $\mathbf{c}$ Flow cytometric analysis of the macrophage phenotypes. $\mathbf{d}$ The gene expression level of macrophages, including iNOS, TNF- $\alpha$, IL-1 $\beta$, TGF- $\beta$, Arg- 1 and IL-10. e Schematic illustration of macrophage-mediated osteogenic differentiation of PDLSCs. $\mathbf{f}$ The immune factors secreted by macrophages in different groups were detected by ELISA. g qRT-PCR analysis for the gene expression levels of ALP, Runx2, OCN and OPN of PDLSCS Cultured in the conditioned medium for 7 days. ${ }^{*} P<0.05,{ }^{* *} P<0.01,{ }^{* * *} P<0.001$
} 
a

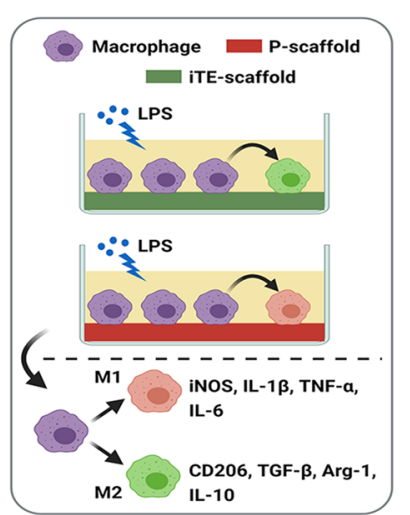

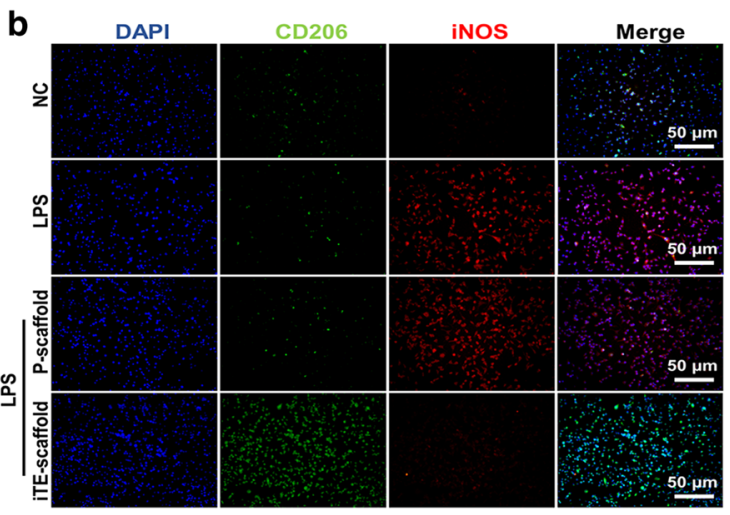

C

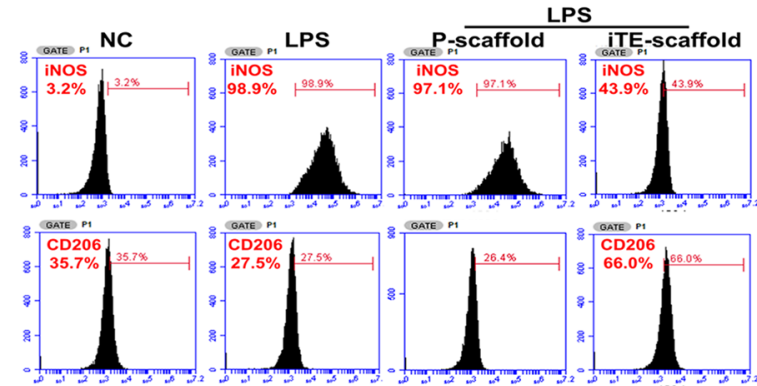

d

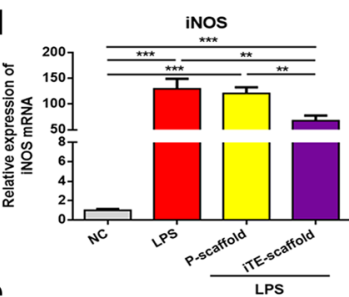

e

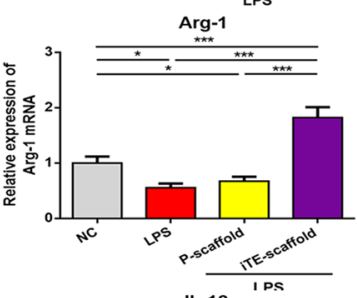

g

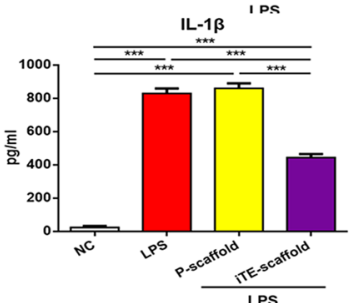

h

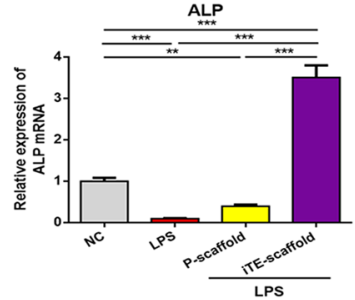

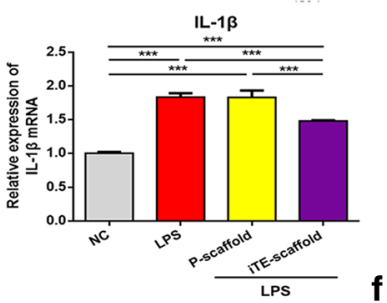
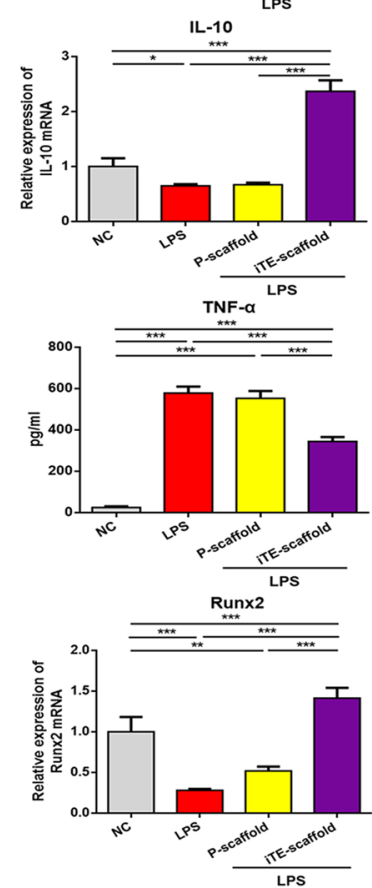
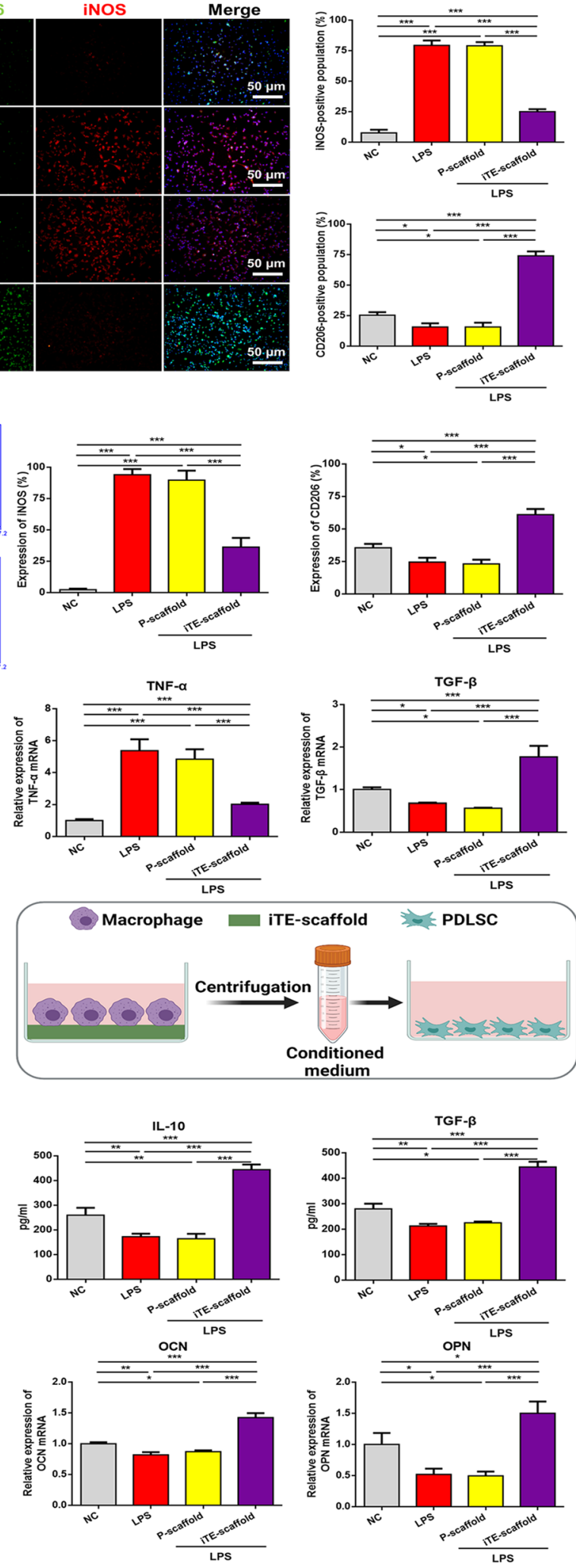

Fig. 4 (See legend on previous page.) 
groups compared to the $\mathrm{NC}$ group $(P<0.001)$, suggesting that the scaffold itself cannot regulate the conversion of the macrophage phenotypes, which was in line with the above results in Fig. 4a-d. Taken together, all above findings demonstrated that M2 polarization of macrophage induced by iTE-scaffold could interact with PDLSCs and promote osteogenic differentiation.

\section{In vivo evaluation of angiogenesis}

The iTE-scaffold was implanted into a rat periodontal bone defect model to study its in vivo bone regenerative capacity (Fig. 5a). Bone, especially alveolar bone, is a highly vascularized tissue. Angiogenesis plays a vital role in bone homeostasis and repair because osteogenesis requires an adequate blood supply to provide oxygen and nutrition [9]. During new blood vessel formation, CD31 is strongly expressed in the endothelium of blood vessels and smooth muscle cells in the walls of blood vessels increasingly express alpha smooth muscle actin ( $\alpha$-SMA) $[30,42]$. Therefore, to investigate the proangiogenic effect of the iTE-scaffold, sections were double-stained for CD31 (red) and $\alpha$-SMA (green). As shown in Fig. 5b, c, at 1 and 2 weeks post-operation, there were lots of CD31 ${ }^{+}$ cells gathering around $\alpha-\mathrm{SMA}^{+}$cells in the defect areas in all groups. As anticipated, significantly more blood vessels formed in iTE-scaffold group. In particular, the microvascular diameter and density were remarkably increased after iTE-scaffold implantation. These in vivo results were consistent with in vitro outcomes, indicating that iTE-scaffold possessed strong capability to promote new blood vessel formation both in vitro and in vivo and was beneficial for bone repair.

\section{In vivo evaluation of immunomodulatory effect}

The interaction between biomaterial and immune system is a key for achieving desired bone repair effect [43]. Among numerous immune cells, macrophages exhibit a vital role in immune defense, and specific macrophage phenotype is crucial for tissue regeneration. The proinflammatory M1 macrophages produce IL- $1 \beta$, TNF- $\alpha$, iNOS, and IL-8 to mediate inflammation, and the antiinflammatory M2 macrophages release pro-healing cytokines, like Arg-1, IL-10, TGF- $\beta$, and IL-4, to maintain tissue homeostasis and facilitate tissue repair [44]. Thus, a smooth and timely shift from pro-inflammatory M1 into pro-healing M2 macrophage is necessary for tissue repair. Accordingly, we subsequently explored the potential effect of iTE-scaffold on macrophage polarization in vivo by double-labeled fluorescence staining of CD68 (a universal macrophage marker), iNOS (an M1 marker), and CD206 (an M2 marker). As displayed in Fig. 5d-f, compared with $\mathrm{NC}$ or P-scaffold groups, there were more $\mathrm{CD} 06^{+} \mathrm{CD}^{+} 8^{+}$cells and fewer iNOS ${ }^{+} \mathrm{CD}^{+} 8^{+}$cells in the iTE-scaffold group at 1 and 2 weeks after implantation $(P<0.05)$. It was worth noting that the number of iNOS ${ }^{+} \mathrm{CD} 8^{+}$cells and $\mathrm{CD} 206^{+} \mathrm{CD}^{+} 8^{+}$cells in P-scaffold group did not show significant difference with that in $\mathrm{NC}$, indicating that scaffold alone did not trigger higher pro-inflammatory response in vivo. These findings were in accordance with above in vitro study, demonstrated that iTE-scaffold could significantly promote the conversion of macrophages from pro-inflammatory M1 type to pro-healing M2 type.

Moreover, M1 and M2 phenotype type macrophages highly influenced the microenvironment of bone repair area via cytokine secretion [35]. Therefore, the expression level of the pro-inflammatory factors (TNF- $\alpha$ and IL-1 $\beta$ ) and the anti-inflammatory factors (IL-10 and TGF- $\beta$ ) within the defective areas were detected by immunohistochemical staining. As shown in Additional file 1: Fig. S4, S5, TNF- $\alpha$ and IL- $1 \beta$ exhibited similar staining results. At week 1 and 2 post-surgery, the optical density (OD) value of TNF- $\alpha$ (Additional file 1: Fig. S4) and IL-1 $\beta$ (Additional file 1: Fig. S5) was remarkably lower in iTE-scaffold group than NC and P-scaffold. It has been reported that IL-1 $\beta$ and TNF- $\alpha$ could also inhibit the synthesis of ALP by osteoblasts and have negative effect on the secretion and mineralization of extracellular bone, which is not conducive to bone repair [45, 46]. Decreased pro-inflammatory factors might contribute to better healing outcome as high pro-inflammatory factors may lead to impaired stem cells and poor regeneration [47]. Besides, the expression levels of IL-1 $\beta$ and TNF- $\alpha$ decreased within time in each group. On the other hand, IL-10 is a pro-healing cytokine, which can inhibit the function of $\mathrm{T}$ helper 1 (Th1) cell and reduce the production of pro-inflammatory cytokines [48]. The expression of IL-10 gradually increased over time, and iTE-scaffold group expressed higher IL-10 than NC and P-scaffold groups (Additional file 1: Fig. S6). Moreover, TGF- $\beta$ is not only the most anti-inflammatory factor, but the upstream of osteogenesis-related BMP signaling [18]. The expression level of TGF- $\beta$ showed similar trends with IL-10 expression (Additional file 1: Fig. S7). In addition, there was no statistical difference in the expression of pro- and anti-inflammatory factors between P-scaffold and NC groups. This finding further indicated that fibrous scaffolds had ideal biocompatibility which was in line with our in vitro study. To sum up, all above results demonstrated that iTE-scaffold could screw macrophage towards pro-healing M2 phenotype and exert antiinflammation effect in vivo, which was in favor of periodontal bone regeneration. 


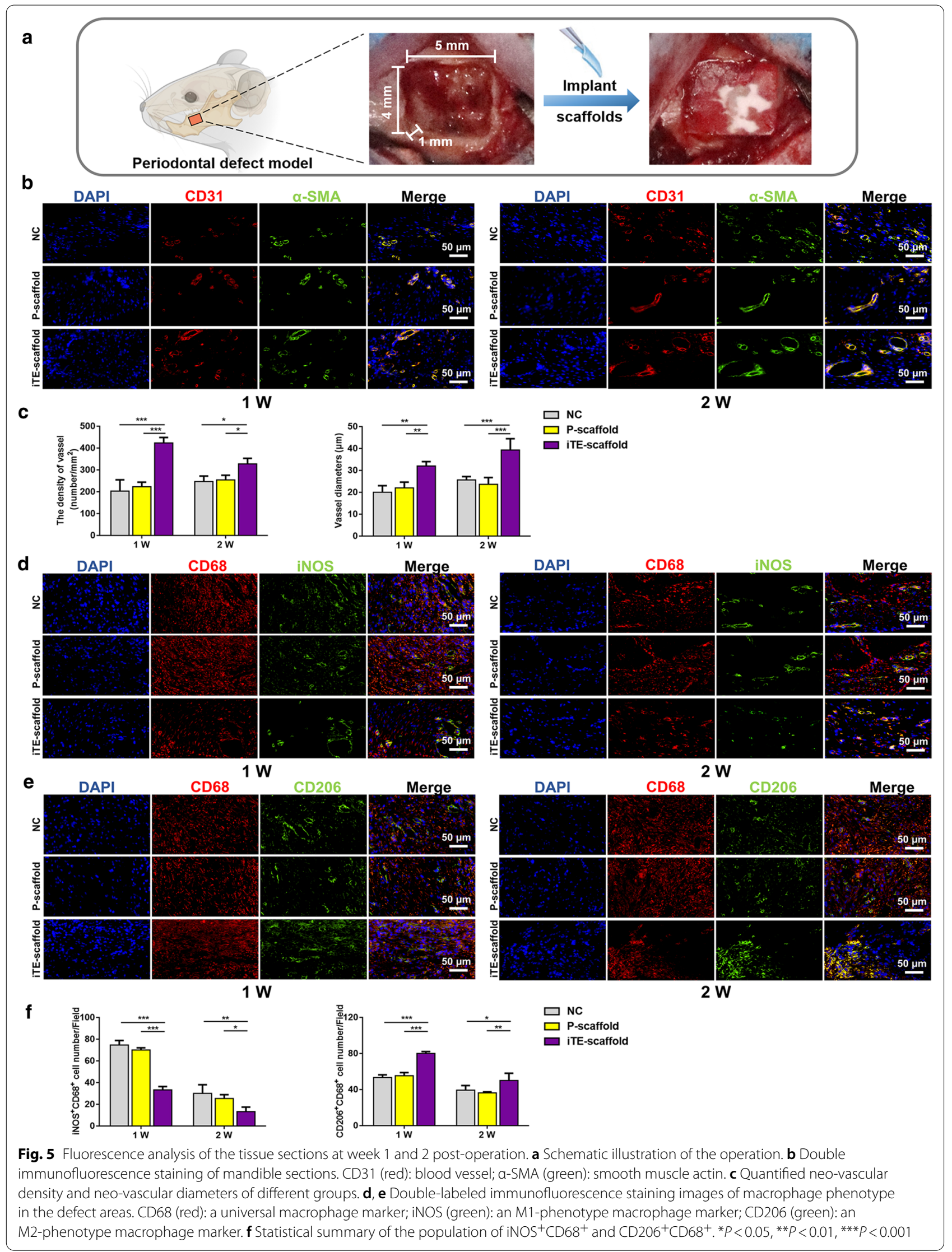




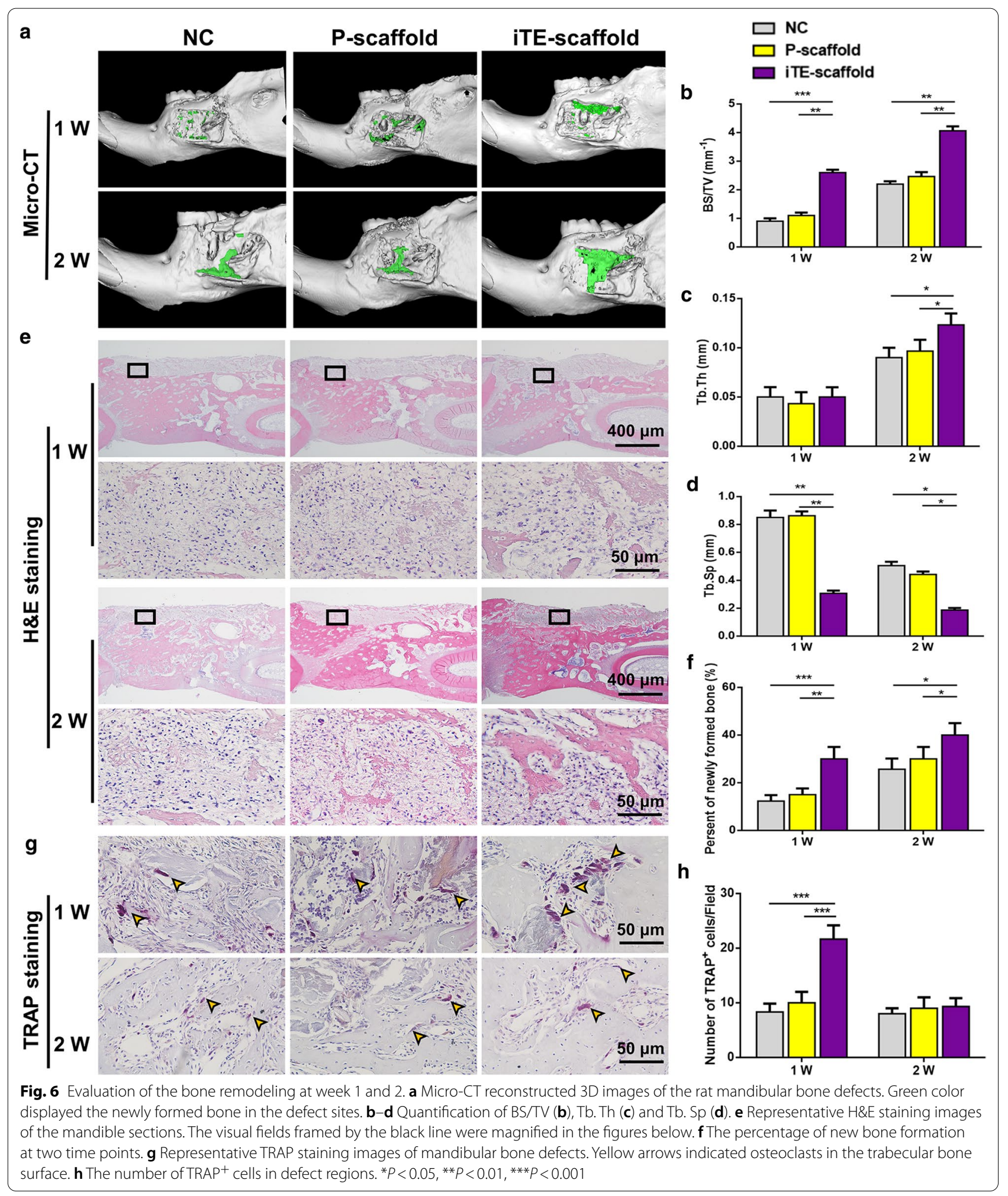




\section{In vivo evaluation of periodontal bone remodeling}

The samples were analyzed with micro-computed tomography (Micro-CT) analysis and quantitative morphometric analysis after 1 and 2 weeks post-operation. The representative images of $3 \mathrm{D}$ digital reconstruction showed that iTE-scaffold presented the largest osteogenic area at all time points (Fig. 6a). Subsequently, we quantitatively and qualitatively characterized the new bone formation in defect areas based on reconstructed 3D Micro-CT images. Significantly, more bone surface (BS) was observed in iTE-scaffold group (Fig. 6b), which was coincident with Micro-CT images. Additionally, the iTE-scaffold group exhibited a greater trabecular bone thickness ( $\mathrm{Tb}$. Th) than $\mathrm{NC}$ and P-scaffold groups at week 2 (Fig. 6c), and simultaneously, as shown in Fig. 6d, the value of trabecular bone separation ( $\mathrm{Tb} . \mathrm{Sp}$ ) reduced over time compared to the other groups. The above finding revealed that more mature and denser bone formed in the iTE-scaffold group.

To further study the periodontal bone regeneration capability of the scaffolds, histological analysis of new bone formation was performed through hematoxylin and eosin (H\&E) staining (Fig. 6e, f). In line with the MicroCT analysis results, a number of new bone formation could be observed in the bone defect areas of iTE-scaffold group, while those in the other groups were barely observed at 1 week after implantation. There were more newly formed bones in the defect areas in all groups at week 2. All the above results proved that iTE-scaffold exhibited remarkable bone repair performance for periodontal bone defect.

In addition, bone remodeling depends on the homeostatic balance between bone formation (osteoblasts) and bone resorption (osteoclasts) [49, 50]. And the initiation and functionalization of osteoclasts play a necessary role in bone remodeling and repair. Thus, the osteoclast activity within the defect areas was evaluated by tartrateresistant acid phosphatase (TRAP) staining. As shown in Fig. $6 \mathrm{~g}, \mathrm{~h}$, the number of $\mathrm{TRAP}^{+}$cells in iTE-scaffold group was over two folds as much as that in NC group at week 1 post-surgery $(P<0.001)$. However, the number of $\mathrm{TRAP}^{+}$cells decreased markedly at week 2 and no significant difference was found among all groups $(P>0.05)$. These results revealed that iTE-scaffold initiated early osteoclastogenesis. Generally, bone apposition occurs at sites where bone resorption has recently occurred, because these sites were prone to attract precursor bone cells and express rich bone matrix proteins for later osteogenic differentiation [50]. Therefore, osteoclastogenesis, even if transient, may be necessary for iTE-scaffold induced anabolism during bone reconstruction.

\section{Conclusions}

In conclusion, we fabricated a novel scaffold based on in situ tissue engineering strategy. The iTE-scaffold was proved for the first time to effectively modulate immune response and angiogenic activity in vitro and in vivo. The iTE-scaffold could significantly steer macrophage polarization towards the pro-healing M2 phenotype and enhance vascularization. Moreover, this immunomodulatory effect could facilitate the osteogenic differentiation of PDLSCs. When the fibrous scaffolds were implanted in the periodontal bone defect model, the iTE-scaffold exhibited an anti-inflammatory response, provided adequate blood supply, and obtained desired bone repair outcome. Collectively, this novel functional iTE-scaffold opens new doors for in situ tissue engineering.

\section{Materials and methods \\ Preparation of the scaffolds}

Poly (lactide-co-glycolide) (LA:GA =50:50) and poly (L-lactic acid) were supplied by Daigang Biomaterial Co., Ltd, Jinan, China. BMP-2 and bFGF were sourced from PeproTech (Rocky Hill, USA). $0.15 \mathrm{~g}$ PLLA and $1 \mathrm{~g}$

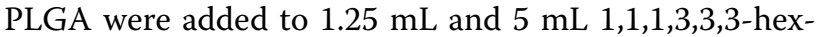
afluoro-2-propanol (HFIP, Macklin, Shanghai, China) so as to make the PLLA core solution and PLGA sheath solution, respectively. After stirring overnight, BMP-2 in phosphate buffered saline (PBS, 0.01 M, HyClone, Logan, USA) $(1 \mu \mathrm{g}, 0.125 \mathrm{~mL})$ was added into the core solution by stirring and sonicating, while bFGF in PBS (500 ng, $0.5 \mathrm{~mL}$ ) was slowly added into the sheath solution in the same way according to our previous study [20]. In addition, the control group selected PBS as the water phase, which was prepared under similar parameters. Then, core and sheath solutions were added into two independent syringes which were connected by a coaxial metal needle. The flow rates of the inner and outer fluids were $0.5 \mathrm{~mL} / \mathrm{h}$ and $2 \mathrm{~mL} / \mathrm{h}$, respectively. And the $15 \mathrm{kV}$ electric potential was used in this study. The electrospinning process was conducted under ambient conditions $\left(25 \pm 2{ }^{\circ} \mathrm{C}\right.$ with relative humidity $\left.50 \pm 5 \%\right)$. Two kinds of scaffolds were fabricated. The scaffold was about $1 \mathrm{~mm}$ in thickness, dominated by the electrospinning time. The control group, representing simple PBS without GFs, was defined as pristine scaffold (P-scaffold) group. And iTEscaffold group denoted the scaffold with bFGF (sheath) and BMP-2 (core). Finally, the collected membranes were preserved at $-20{ }^{\circ} \mathrm{C}$ for future experiments.

\section{Characterizations of the scaffolds}

The surface morphology and structure of the scaffolds was acquired under SEM (S-4800, Hitachi, Tokyo, Japan). To corroborate the shell/core structure of fibrous scaffold, the fluorescence dyes Rhodamine B (red) and 
Coumarin 6 (green) (Sigma-Aldrich, USA) were subsequently added into core and shell solutions, respectively. Afterwards, the distribution of fluorescence dyes in collected fibers was observed by a fluorescence microscope (Leica, Wetzlar, German). The chemical composition of PLLA and PLGA mixture, P-scaffold and iTE-scaffold was evaluated by the FTIR spectroscopy (Thermo Fisher Scientific, Waltham, USA). The mechanical properties of scaffolds were characterized utilizing universal testing machine (Instron, Boston, USA). The surface wettability of the scaffolds was assessed using a pendant drop method on the WCA analyzer (DSA10, Kruss, Hamburg, Germany).

\section{Cell culture}

The isolation of human PDLSCs was carried out based on our previously procedures [51]. Primary PDLSCs were maintained in DMEM (HyClone) containing 20\% FBS (BioInd, Kibbutz, Israel). PDLSCs were passaged using trypsin-EDTA solution (HyClone) until 90\% confluent cells were reached. Afterwards, the passaged cells were cultivated with $10 \%$ FBS in DMEM.

RAW264.7 macrophages were sourced from the Shanghai Cell Bank of the Chinese Academy of Sciences and cultivated in 10\% FBS-supplemented DMEM. HUVECs were commercially purchased (ScienCell, San Diego, USA) and seeded in supplemented endothelial culture medium (ECM, ScienCell). All cells were cultured at a $37{ }^{\circ} \mathrm{C}$ and $5 \% \mathrm{CO}_{2}$ incubator with a humidified atmosphere.

\section{Cell viability evaluation}

The compatibility of PDLSCs seeded on tissue culture plate, P-scaffold and iTE-scaffold was evaluated by a LIVE/DEAD viability/cytotoxicity kit (Invitrogen, CA, USA). Cells cultured on the tissue culture plate were served as NC. After incubation for $24 \mathrm{~h}$, propidium iodide (PI) and calcein acetoxymethyl ester (AM) were used to stain the dead and live cells, respectively. Afterwards, the samples were observed under the fluorescence microscope. Meanwhile, cell adhesion assay of the fibrous scaffolds was performed as previously reported [52]. Cell viability and proliferation on different substrates were evaluated by CCK-8 (Dojindo Laboratories, Tokyo, Japan) after cultivated for $24 \mathrm{~h}$. In addition, RAW264.7 macrophages were seeded on the scaffolds. And the morphology of macrophages on P-scaffold and iTE-scaffold was characterized with an SEM after dehydration by gradient alcohol.

\section{In vitro vascularization assay}

PDLSCs were cultured with EGM-2 (Lonza, Walkersville, USA) on different membranes for 7 days. In vitro angiogenic capacity was assessed by the tube formation assay on Matrigel (354230, Corning, NY, USA) as before [48]. PDLSCs in EGM-2, PDLSCs on P-scaffold, PDLSCs on iTE-scaffold and HUVECs were collected and resuspended in the medium of EGM-2. Then, cell suspensions $\left(5 \times 10^{4}\right.$ cells $)$ in $100 \mu \mathrm{l} \mathrm{EGM-2}$ were reseeded onto Matrigel-coated wells. PDLSCs cultured in EGM-2 regarded as the $\mathrm{NC}$, while HUVECs resuspended in EGM-2 regarded as the positive control. Tube formation was observed at predetermined time points $(12 \mathrm{~h}, 24 \mathrm{~h})$ under the inverted light microscope (Olympus, Tokyo, Japan). Subsequently, the following angiogenic parameters, including total length, the number of junctions, meshes and nodes were evaluated by Image J software (NIH, USA).

The relative gene expression levels of angiogenic differentiation (CD31, VEGF, PLGF and SCF) were analyzed by qRT-PCR. And Glyceraldehyde-3-phosphate dehydrogenase $(G A P D H)$ primer was selected as the housekeeping gene and primer sequences were provided in Additional file 1: Table S1.

In addition, for fluorescence analysis, the samples were fixed with paraformaldehyde (4\%) and blocked with normal goat serum for $40 \mathrm{~min}$. Thereafter, samples were incubated with rabbit anti-CD31 (1:200, ab32457, Abcam, Cambridge, UK) overnight. The samples were further incubated with CoraLite488-conjugated goat anti-rabbit secondary antibody (1:800, SA00013-2, Proteintech, Chicago, IL, USA) and then counterstained with DAPI (ab104139, Abcam). The images were captured with the fluorescence microscope (Olympus).

\section{In vitro osteoimmunomodulatory function characterization Inflammatory response of macrophages}

RAW264.7 macrophages were collected, counted, and resuspended in the inflammatory induction medium [10\% FBS DMEM supplemented with $100 \mathrm{ng} / \mathrm{mL}$ LPS (Sigma-Aldrich)] according to a previous study [37]. Subsequently, cell suspensions $\left(2 \times 10^{5}\right.$ cells $)$ were seeded onto the P-scaffold and iTE-scaffold within each well in 6-well plates. Moreover, RAW264.7 cultivated in 10\% FBS in DMEM without LPS denoted as the NC group, while those cultured in the inflammatory induction medium served as the LPS group. The induction medium was collected after cultivation for $24 \mathrm{~h}$ and replaced with $6 \mathrm{~mL}$ DMEM. After cultivation for $6 \mathrm{~h}$, the corresponding medium was collected as the conditioned medium for further experiments. Subsequently, the macrophages were double-stained with primary antibodies of antimannose receptor antibody (1:300, ab64693, Abcam) and iNOS antibody (1:200, ab49999, Abcam). The fluorescence images were obtained under the fluorescence 
microscope. Meanwhile, the expression of M1/M2 macrophage surface markers was assessed by flow cytometry (Accuri-C6, BD Biosciences, San Diego, USA). Stimulated macrophages were incubated with allophycocyanin (APC)-conjugated anti-iNOS (2102823, Invitrogen) and anti-CD206 (MMR) (141707, Biolegend). Related conjugated IgG was used as control. Subsequently, the gene level was measured by qRT-PCR. The detected genes included TNF- $\alpha$, iNOS, Arg-1, CD206, IL-10, IL-1 and TGF- $\beta$. And the primer sequences were showed in Additional file 1: Table S2. Besides, the concentration of anti- and pro- inflammatory cytokines (IL-10, TGF- $\beta$, IL- $1 \beta$ and TNF- $\alpha$ ) in collected induction medium were detected with corresponding ELISA kits (Biolegend) based on the manufacturer's guidance.

\section{Osteogenic differentiation of PDLSCs}

To assess whether macrophages in response to membranes could regulate osteogenic differentiation of PDLSCs. Cells $\left(2 \times 10^{5}\right.$ cells per well $)$ were seeded into the 6-well plate and stimulated with conditioned medium to mimic the immune environment in vivo. After cultured for 7 days, the osteogenesis-related genes in PDLSCs were evaluated by qRT-PCR. The sequences of the primers of $A L P, \operatorname{Run} x 2, O C N$ and $O P N$ were showed in the Additional file 1: Table S3.

\section{Establishment of mandibular periodontal bone defect model}

A total of eighteen eight-week-old male Wistar rats were used in this study. The rats were randomly divided into NC, P-scaffold, and iTE-scaffold groups. Surgical procedures were conducted as previously described [53]. In brief, after shaving and cleaning, the buccal surface of the mandible was exposed. Then, a $5 \times 4 \times 1 \mathrm{~mm}$ defect was created by a fissure bur. The defect area located about $1 \mathrm{~mm}$ behind the front border of the mandible and $1 \mathrm{~mm}$ below the upper border of the mandible. Subsequently, the defects were filled with iTE-scaffold and P-scaffold based on the respective groups, whereas the defect areas left untreated defined as the NC group. The rats were euthanized at week 1 and 2 post-surgery, the samples were harvested and fixed for the following analysis.

\section{Micro-CT analysis}

To evaluate the bone regeneration in the defect area, the harvested mandibles were scanned by high-resolution Micro-CT (PerkinElmer, MA, USA). Samples were reconstructed and analyzed using supporting software. The various bone parameters, including BS/TV (\%), Tb. $\mathrm{Sp}(\mathrm{mm})$ and $\mathrm{Tb}$. Th $(\mathrm{mm})$ were analyzed.
Histology, immunohistochemistry (IHC) and immunofluorescence analyses

The samples were decalcified in $10 \%$ disodium ethylenediaminetetraacetic acid (EDTA- $\mathrm{Na}_{2}$, Solarbio, Beijing, China) at $4{ }^{\circ} \mathrm{C}$ for 30 days. Afterwards, decalcified samples were longitudinally embedded in paraffin wax and sliced into $5 \mu \mathrm{m}$ sections. Then, the tissue sections were stained with H\&E staining kit (Solarbio) and TRAP staining kit (Solarbio) based on the manufacturer's instructions. The stained sections were photographed under the microscope (BX53, Olympus) and analyzed with Image ProPlus 6.0 Software (Media Cybernetics, Silver Spring, USA).

IHC staining was performed with an anti-Rabbit HRP/DAB Detection Kit (ab64261, Abcam) following the manufacturer's protocol. The primary antibodies used anti-TNF- $\alpha$ (1:200, AF7014, Affinity, OH, USA), anti-IL-1 $\beta$ (1:200, AF5103, Affinity), anti-IL-10 (1:300, DF6894, Affinity), TGF- $\beta$ (1:200, AF1027, Affinity). The images were photographed under the microscope. For semiquantitative analysis, mean OD of IL-10, TNF- $\alpha$, IL- $1 \beta$ and TGF- $\beta$ was measured by Image Pro-Plus 6.0 software.

Immunofluorescent staining was performed as described before [54]. In order to detect phenotype switching of macrophages and angiogenesis in vivo, the tissue sections were incubated with primary antibodies of anti-CD68 (1:250, ab955, Abcam)/anti-iNOS (1:200, ab15323, Abcam), anti-CD68/anti-CD206 and anti- $\alpha$-SMA (1:400, ab7817, Abcam)/anti-CD31 (1:300, ab28364, Abcam). The fluorescent slides were finally covered by the cover glass using mounting medium containing DAPI. The fluorescent images were obtained with the fluorescence microscope, and the number of $\mathrm{CD}^{206}{ }^{+} \mathrm{CD} 8^{+}$cells, $\mathrm{iNOS}^{+} \mathrm{CD}^{+}{ }^{+}$cells, $\alpha-\mathrm{SMA}^{+}$cells and $\mathrm{CD}_{3}{ }^{+}$cells in the defects were counted and measured by Image J software.

\section{Statistical analysis}

All statistical data were shown as mean \pm standard deviation (SD). Tests were analyzed using GraphPad Prism software (Version 6, Boston, USA). Differences between two groups were performed by Student's t-test, and multiple group comparisons were performed by one-way or two-way ANOVA. And Turkey HSD comparison test was utilized when multiple comparisons were performed. Differences were considered to be significant at $P<0.05$.

\footnotetext{
Abbreviations

iTE-scaffold: In situ tissue engineering scaffold; P-scaffold: Pristine scaffold; PLGA: Poly (lactide-co-glycolide); PLLA: Poly (L-lactic acid); bFGF: Basic fibroblast growth factor; BMP-2: Bone morphogenetic protein-2; PDLSC:
} 
Periodontal ligament stem cell; HUVEC: Human umbilical vein endothelial cell; LPS: Lipopolysaccharide; SEM: Scanning electron microscopy; FTIR: Fourier transform infrared spectroscopy; WCA: Water contact angle; DMEM: Dulbecco's modified Eagle's medium; ECM: Endothelial culture medium; VEGF: Vascular endothelial growth factor; PLGF: Placental growth factor; SCF: Stem cell factor; GAPDH: Glyceraldehyde-3-phosphate dehydrogenase; qRT-PCR: Quantitative real-time polymerase chain reaction; iNOS: Inducible nitric oxide synthase; TGF- $\beta$ : Transforming growth factor beta; TNF- $\alpha$ : Tumor necrosis factor-a; Arg-1: Arginase-1; IL-10/1B: Interleukin-10/1 beta; ELISA: Enzymelinked immunosorbent assay; ALP: Alkaline phosphatase; Runx2: Runt-related transcription factor 2; OCN: Osteocalcin; OPN: Osteopontin; Micro-CT: Microcomputed tomography; BS/TV: Bone surface area/total volume; Tb. Sp: Trabecular bone separation; Tb. Th: Trabecular bone thickness; H\&E: Hematoxylin and eosin; TRAP: Tartrate-resistant acid phosphatase; IHC: Immunohistochemistry; Fig.: Figure.

\section{Supplementary Information}

The online version contains supplementary material available at https://doi. org/10.1186/s12951-021-00992-4.

Additional file 1: Table S1. Primer sequences for qRT-PCR. Table S2. Primer sequences for qRT-PCR. Table S3. Primer sequences for GRT-PCR. Fig. S1. Fluorescence microscopy images of the fibrous scaffolds after fluorescent staining. Fig. S2. WCA of different fibrous membranes. Fig. S3. SEM images of macrophages cultured on P-scaffold and iTE-scaffold for 24 h. Fig. S4. Immunohistochemistry analysis of TNF-a expression in all groups. Fig. S5. Immunohistochemistry analysis of IL-1 $\beta$ expression in all groups. Fig. S6. Immunohistochemistry analysis of IL-10 expression in all groups. Fig. S7. Immunohistochemistry analysis of TGF- $\beta$ expression in all groups.

\section{Acknowledgements}

All schematic diagrams were created with Biorender.com.

\section{Authors' contributions}

TD performed the experiments and wrote the manuscript. WYK and JHL analyzed the data. LY helped with the in vivo experiments. SHG supervised the project and was responsible for overall study direction. All authors read and approved the final manuscript.

\section{Funding}

This research was supported by the National Natural Science Foundation of China (No. 81873716), The Construction Engineering Special Fund of "Taishan Scholars" of Shandong Province (No. ts20190975 and tsqn201909180), Shandong Provincial Natural Science Foundation (No. ZR2020QH159), Collaborative Innovation Center of Technology and Equipment for Biological Diagnosis and Therapy in Universities of Shandong, The National Key Research and Development Program of China (No. 2017YFA0104604). The funders had no role in study design, data collection and analysis, decision to publish or preparation of the manuscript.

\section{Availability of data and materials}

The datasets in the current study are included in the published article or available from the corresponding author on reasonable request.

\section{Declarations}

\section{Ethics approval and consent to participate}

All experimental procedures were approved by the School and Hospital of Stomatology, Shandong University (Protocol Number: GR201801 and GD201801).

\section{Consent for publication}

Not applicable.

\section{Competing interests}

The authors declare no competing financial interest.
Received: 21 June 2021 Accepted: 8 August 2021

Published online: 17 August 2021

\section{References}

1. Slots J. Periodontitis: facts, fallacies and the future. Periodontol. 2000;2017(75):7-23.

2. Kinane DF, Stathopoulou PG, Papapanou PN. Periodontal diseases. Nat Rev Dis Primers. 2017;3:17038.

3. Cardoso EM, Reis C, Manzanares-Cespedes MC. Chronic periodontitis, inflammatory cytokines, and interrelationship with other chronic diseases. Postgrad Med. 2018;130:98-104.

4. Bosshardt DD, Sculean A. Does periodontal tissue regeneration really work? Periodontol. 2000;2009(51):208-19.

5. Deschaseaux F, Sensebe L, Heymann D. Mechanisms of bone repair and regeneration. Trends Mol Med. 2009;15:417-29.

6. Hu C, Chu C, Liu L, Wang C, Jin S, Yang R, Rung S, Li J, Qu Y, Man Y. Dissecting the microenvironment around biosynthetic scaffolds in murine skin wound healing. Sci Adv. 2021;7:eabf0787.

7. Wynn TA, Vannella KM. Macrophages in tissue repair, regeneration, and fibrosis. Immunity. 2016;44:450-62.

8. Hankenson KD, Dishowitz M, Gray C, Schenker M. Angiogenesis in bone regeneration. Injury. 2011:42:556-61.

9. Ramasamy SK, Kusumbe AP, Schiller M, Zeuschner D, Bixel MG, Milia C, Gamrekelashvili J, Limbourg A, Medvinsky A, Santoro MM, et al. Blood flow controls bone vascular function and osteogenesis. Nat Commun 2016:7:13601.

10. Lee JS, Jin Y, Park HJ, Yang K, Lee MS, Yang HS, Cho SW. In situ bone tissue engineering with an endogenous stem cell mobilizer and osteoinductive nanofibrous polymeric scaffolds. Biotechnol J. 2017:12:1700062.

11. Wu R-X, Xu X-Y, Wang J, He X-T, Sun H-H, Chen F-M. Biomaterials for endogenous regenerative medicine: coaxing stem cell homing and beyond. Appl Mater Today. 2018;11:144-65.

12. LiX. The FGF metabolic axis. Front Med. 2019:13:511-30.

13. Presta M, Foglio E, Churruca Schuind A, Ronca R. Long pentraxin-3 modulates the angiogenic activity of fibroblast growth factor-2. Front Immunol. 2018;9:2327.

14. Sahni A, Francis CW. Stimulation of endothelial cell proliferation by FGF- 2 in the presence of fibrinogen requires alphavbeta3. Blood. 2004; 104:3635-41.

15. Schmidt A, Ladage D, Schinkothe T, Klausmann U, Ulrichs C, Klinz FJ, Brixius K, Arnhold S, Desai B, Mehlhorn U, et al. Basic fibroblast growth factor controls migration in human mesenchymal stem cells. Stem Cells. 2006;24:1750-8.

16. Pan X, Xu S, Zhou Z, Wang F, Mao L, Li H, Wu C, Wang J, Huang Y, Li D, et al. Fibroblast growth factor-2 alleviates the capillary leakage and inflammation in sepsis. Mol Med. 2020;26:108.

17. Fujihara C, Kanai Y, Masumoto R, Kitagaki J, Matsumoto M, Yamada S, Kajikawa T, Murakami S. Fibroblast growth factor-2 inhibits CD40-mediated periodontal inflammation. J Cell Physiol. 2019;234:7149-60.

18. Chen G, Deng C, Li YP. TGF-beta and BMP signaling in osteoblast differentiation and bone formation. Int J Biol Sci. 2012;8:272-88.

19. Chung VH, Chen AY, Kwan CC, Chen PK, Chang SC. Mandibular alveolar bony defect repair using bone morphogenetic protein 2-expressing autologous mesenchymal stem cells. J Craniofac Surg. 2011;22:450-4.

20. Ding T, Li J, Zhang X, Du L, Li Y, Li D, Kong B, Ge S. Super-assembled core/ shell fibrous frameworks with dual growth factors for in situ cementumligament-bone complex regeneration. Biomater Sci. 2020;8:2459-71.

21. Kang W, Liang Q, Du L, Shang L, Wang T, Ge S. Sequential application of bFGF and BMP-2 facilitates osteogenic differentiation of human periodontal ligament stem cells. J Periodontal Res. 2019;54:424-34.

22. Li Y, Sun XS. Preparation and characterization of polymer-inorganic nanocomposites by in situ melt polycondensation of L-lactic acid and surface-hydroxylated MgO. Biomacromol. 2010;11:1847-55.

23. Chazaud B. Macrophages: supportive cells for tissue repair and regeneration. Immunobiology. 2014;219:172-8.

24. Poologasundarampillai G, Yu B, Jones JR, Kasuga T. Electrospun silica/ PLLA hybrid materials for skeletal regeneration. Soft Matter. 2011;7:10241.

25. He P, Zhong Q, Ge Y, Guo Z, Tian J, Zhou Y, Ding S, Li H, Zhou C. Dual drug loaded coaxial electrospun PLGA/PVP fiber for guided tissue 
regeneration under control of infection. Mater Sci Eng C Mater Biol Appl. 2018;90:549-56.

26. Hotchkiss KM, Reddy GB, Hyzy SL, Schwartz Z, Boyan BD, OlivaresNavarrete R. Titanium surface characteristics, including topography and wettability, alter macrophage activation. Acta Biomater. 2016;31:425-34

27. Davenport Huyer L, Pascual-Gil S, Wang Y, Mandla S, Yee B, Radisic M. Advanced strategies for modulation of the material-macrophage interface. Adv Func Mater. 2020;30:1909331.

28. Takehara N, Tsutsumi Y, Tateishi K, Ogata T, Tanaka H, Ueyama T, Takahashi T, Takamatsu T, Fukushima M, Komeda M, et al. Controlled delivery of basic fibroblast growth factor promotes human cardiosphere-derived cell engraftment to enhance cardiac repair for chronic myocardial infarction. J Am Coll Cardiol. 2008;52:1858-65.

29. Horio T, Fujita M, Tanaka Y, Ishihara M, Kishimoto S, Nakamura S, Hase K, Maehara T. Efficacy of fragmin/protamine microparticles containing fibroblast growth factor-2 (F/P MPs/FGF-2) to induce collateral vessels in a rabbit model of hindlimb ischemia. JVasc Surg. 2011;54:791-8.

30. Lertkiatmongkol P, Liao D, Mei H, Hu Y, Newman PJ. Endothelial functions of platelet/endothelial cell adhesion molecule-1 (CD31). Curr Opin Hematol. 2016:23:253-9.

31. Michiels C. Endothelial cell functions. J Cell Physiol. 2003;196:430-43.

32. Liu Y, Liu P, Song Y, Li S, Shi Y, Quan K, Yu G, Li P, An Q, Zhu W. A heparinrosuvastatin-loaded $P(L L A-C L)$ nanofiber-covered stent inhibits inflammatory smooth-muscle cell viability to reduce in-stent stenosis and thrombosis. J Nanobiotechnology. 2021;19:123.

33. Bai L, Du Z, Du J, Yao W, Zhang J, Weng Z, Liu S, Zhao Y, Liu Y, Zhang X, et al. A multifaceted coating on titanium dictates osteoimmunomodulation and osteo/angio-genesis towards ameliorative osseointegration. Biomaterials. 2018;162:154-69.

34. Spiller KL, Nassiri S, Witherel CE, Anfang RR, Ng J, Nakazawa KR, Yu T, Vunjak-Novakovic G. Sequential delivery of immunomodulatory cytokines to facilitate the M1-to-M2 transition of macrophages and enhance vascularization of bone scaffolds. Biomaterials. 2015;37:194-207.

35. Wu J, Xiao Z, Chen A, He H, He C, Shuai X, Li X, Chen S, Zhang Y, Ren B, et al. Sulfated zwitterionic poly(sulfobetaine methacrylate) hydrogels promote complete skin regeneration. Acta Biomater. 2018;71:293-305.

36. Pajarinen J, Lin T, Gibon E, Kohno Y, Maruyama M, Nathan K, Lu L, Yao Z, Goodman SB. Mesenchymal stem cell-macrophage crosstalk and bone healing. Biomaterials. 2019;196:80-9.

37. Liu L, Guo H, Song A, Huang J, Zhang Y, Jin S, Li S, Zhang L, Yang C, Yang P. Progranulin inhibits LPS-induced macrophage M1 polarization via NF-кB and MAPK pathways. BMC Immunol. 2020:21:32.

38. Sharma U, Pal D, Prasad R. Alkaline phosphatase: an overview. Indian J Clin Biochem. 2014;29:269-78.

39. Komori T. Runx2, an inducer of osteoblast and chondrocyte differentiation. Histochem Cell Biol. 2018:149:313-23.

40. Morrison SJ, Scadden DT. The bone marrow niche for haematopoietic stem cells. Nature. 2014;505:327-34.

41. Greenbaum A, Hsu YM, Day RB, Schuettpelz LG, Christopher MJ, Borgerding JN, Nagasawa T, Link DC. CXCL12 in early mesenchymal progenitors is required for haematopoietic stem-cell maintenance. Nature. 2013:495:227-30.

42. Sun Y, Yang Z, Zheng B, Zhang X-H, Zhang M-I, Zhao X-s, Zhao H-y, Suzuki T, Wen J-k. A novel regulatory mechanism of smooth muscle $a$-actin expression by NRG-1/circACTA2/miR-548f-5p axis. Circ Res. 2017;121:628-35.
43. Gan J, Dou Y, Li Y, Wang Z, Wang L, Liu S, Li Q, Yu H, Liu C, Han C, et al. Producing anti-inflammatory macrophages by nanoparticle-triggered clustering of mannose receptors. Biomaterials. 2018;178:95-108.

44. Olefsky JM, Glass CK. Macrophages, inflammation, and insulin resistance. Annu Rev Physiol. 2010;72:219-46.

45. Einhorn TA, Gerstenfeld LC. Fracture healing: mechanisms and interventions. Nat Rev Rheumatol. 2015;11:45-54.

46. Abdelmagid SM, Barbe MF, Safadi FF. Role of inflammation in the aging bones. Life Sci. 2015:123:25-34.

47. Wang L, Zhao Y, Liu Y, Akiyama K, Chen C, Qu C, Jin Y, Shi S. IFN- $Y$ and TNF-a synergistically induce mesenchymal stem cell impairment and tumorigenesis via NFKB signaling. STEM CELLS. 2013;31:1383-95.

48. Yi B, Ding T, Jiang S, Gong T, Chopra H, Sha O, Dissanayaka WL, Ge S, Zhang C. Conversion of stem cells from apical papilla into endothelial cells by small molecules and growth factors. Stem Cell Res Ther. 2021;12:266.

49. Ottewell PD. The role of osteoblasts in bone metastasis. J Bone Oncol. 2016:5:124-7.

50. Detsch R, Boccaccini AR. The role of osteoclasts in bone tissue engineering. J Tissue Eng Regen Med. 2015;9:1133-49.

51. Du L, Feng R, Ge S. PTH/SDF-1alpha cotherapy promotes proliferation, migration and osteogenic differentiation of human periodontal ligament stem cells. Cell Prolif. 2016;49:599-608.

52. Zhao Q, Wang J, Cui H, Chen H, Wang Y, Du X. Programmed shapemorphing scaffolds enabling facile 3D endothelialization. Adv Funct Mat 2018;28:1801027.

53. Liang Q, Du L, Zhang R, Kang W, Ge S. Stromal cell-derived factor-1/ Exendin-4 cotherapy facilitates the proliferation, migration and osteogenic differentiation of human periodontal ligament stem cells in vitro and promotes periodontal bone regeneration in vivo. Cell Prolif. 2021;54:12997.

54. Hu Z, Ma C, Rong X, Zou S, Liu X. Immunomodulatory ECM-like microspheres for accelerated bone regeneration in diabetes mellitus. ACS Appl Mater Interfaces. 2018;10:2377-90.

\section{Publisher's Note}

Springer Nature remains neutral with regard to jurisdictional claims in published maps and institutional affiliations.

Ready to submit your research? Choose BMC and benefit from

- fast, convenient online submission

- thorough peer review by experienced researchers in your field

- rapid publication on acceptance

- support for research data, including large and complex data types

- gold Open Access which fosters wider collaboration and increased citations

- maximum visibility for your research: over $100 \mathrm{M}$ website views per year

At BMC, research is always in progress.

Learn more biomedcentral.com/submissions 